\title{
A Patch Model for the Transmission Dynamics of Zika Virus from Rio de Janeiro to Miami during Carnival and the Olympics
}

\author{
Caitlin Oleson \\ College of Engineering \\ University of Nevada, Reno \\ cjoleson@gmail.com \\ Adviser: \\ Marc Artzrouni \\ Department of Mathematics \\ University of Pau and Pays de l'Adour \\ marc.artzrouni@univ-pau.fr
}

\begin{abstract}
Since late 2015 there has been an outbreak of Zika virus disease in South and Central America. This is particularly alarming because of its connection to microcephaly in infants born to mothers who were infected while pregnant. There are two mass gatherings which happened in 2016 in Brazil: Carnival (February 6-10 2016) and the Olympics (August 5-21, 2016). These events brought large groups of foreigners to Brazil who could have been exposed to the virus and transported it back to their home country. We created a mathematical model to analyze if a group of visitors to Rio de Janeiro for either of these events would cause an outbreak in Miami, Florida when they went home. Our model shows that if conditions are assumed to be the same for the populations in Miami and Rio de Janeiro, the visitors to Carnival could cause an outbreak in Miami in October that in three months infects roughly $75 \%$ of the population. If, however, the parameters for the model are modified to reflect different lifestyles and mosquito populations in Miami, the size of the outbreak there can be reduced. The model for Rio de Janeiro suggests that by August the majority of the population will already have been infected, hence immune, and there will be a low number of mosquitoes. Therefore, our model predicts that due to reduced infection rates during the Olympics the chance of visitors bringing back the disease to Miami is very low.
\end{abstract}




\section{Introduction}

The vector-borne Zika virus has caused widespread health problems in 2015 and 2016 in South America. It is spread by members of the Aedes family of mosquitoes, the same ones that transmit dengue and chikungunya. First discovered in 1947 in Uganda, it has caused multiple outbreaks throughout the 21st century [14]. In 2014 there were over 30,000 reported cases in French Polynesia and it was estimated that up to $94 \%$ of the population there had been infected [14]. The most recent outbreak began in 2015 in Brazil and was officially

labeled a Public Health Emergency of International Concern by the World Health Organization (WHO) on February 1, 2016 [4]. While the disease can be asymptomatic or cause minor fever and a rash, the virus has also been linked to Guillian-Barré Syndrome and microcephaly in infants born to mothers infected during pregnancy. Between September 2015 and July 2016 there have been over 225,000 suspected and reported cases of Zika virus disease and 1,638 confirmed cases of microcephaly associated with Zika in Brazil [17].

Recently there has been interest in the public health implications of mass gatherings [24]. There were two such gatherings during 2016 which brought large numbers of foreigners to Brazil: Carnival and the Olympics. Carnival occurred between February 6 and 10, where up to 500,000 foreigners went to Brazil to participate in the festivities [20]. The 2016 Summer Olympics took place August 5-21 in Rio de Janeiro, where upwards of 500,000 people came from across the globe to view them [19]. The influx of people for either of these events brought a new susceptible population to Brazil who, if infected, could bring the Zika virus back to their home country. Due to the widespread habitat of the mosquitoes that can transmit the virus it is possible that Carnival or the Olympics will further the global spread of Zika and begin new outbreaks in other countries. Indeed, 150 health professionals wrote a letter to WHO calling for the Olympics to be moved or postponed due to these concerns [1]. WHO dismissed the letter saying such claims were unfounded [23]. Our goal is to use mathematical modeling to help shed light on this issue and investigate the public health implications of visitors to either Carnival or the Olympics.

The model consists of a two-patch system to demonstrate the possible spread of Zika virus by visitors from Miami who went to Rio de Janeiro for Carnival or the Olympics. The first patch is in Rio de Janeiro. The second patch was placed in Miami because there is a high mosquito population, high human population, and there is more movement from Miami to Central and South America than from other U.S. cities [10].

In Section 2 we define the compartmental model for the human and mosquito populations and the mechanisms which drive the transmission of the virus between humans and mosquitoes. In Section 3 we specify parameter values and explore the transmission data for the two mass gatherings of interest. We summarize results in the Discussion and Conclusion section. 


\section{Model}

Mathematical modeling of diseases has been used for the past century to provide insights on disease spread and transmission dynamics. The first model was created in 1927 and called the Kermack-McKendrick Model [11]. This simple model consisted of three compartments: susceptible, infected, and recovered. There have been multiple variations on this model since its introduction to include an exposed category or the transmission between humans and mosquitoes $[14,16]$. The model we used (Figure 1) to express the transmission of the Zika virus between humans and mosquitoes consists of seven compartments for each of the two patches: Rio de Janeiro (indexed by $i=1$ ) and Miami $(i=2)$. Our model is based on those in [14] and [16]. The four human compartments are i) the susceptible (notated as $S_{H}^{i}(t)$ in Patch $i$ at time $t$ ) representing those who can contract the disease; ii) the exposed $\left(E_{H}^{i}(t)\right)$ are the humans incubating the disease but not yet spreading it; iii) the infected $\left(I_{H}^{i}(t)\right)$ are the humans who can spread the disease but may or may not show symptoms; iv) the recovered $\left(R_{H}^{i}(t)\right)$ are the recovered humans. The sum of these populations is $N_{H}^{i}(t)$. We assume that once recovered, a person is immune and cannot be re-infected.

The three mosquito compartments are susceptible $\left(S_{V}^{i}(t)\right)$, exposed $\left(E_{V}^{i}(t)\right)$, and infected $\left(I_{V}^{i}(t)\right)$, with no recovered compartment because we assume an infected mosquito remains so until its death. The sum of these populations is $N_{V}^{i}(t)$. Infections between humans and mosquitoes occurs either when an infected human is bitten by a susceptible mosquito or when an infected mosquito bites a susceptible human (Figure 2). We made an assumption of homogeneous mixing in both human and mosquito populations. If for convenience we drop the dependence on $t$, the model can be expressed as a system of differential equations:

$$
\begin{aligned}
\frac{d S_{H}^{i}}{d t} & =\nu_{H}^{i} N_{H}^{i}-\lambda_{H}^{i}\left(N_{V}^{i} / N_{H}^{i}\right) S_{H}^{i}-\mu_{H}^{i} S_{H}^{i} \\
\frac{d E_{H}^{i}}{d t} & =\lambda_{H}^{i}\left(N_{V}^{i} / N_{H}^{i}\right) S_{H}^{i}-\alpha_{H}^{i} E_{H}^{i}-\mu_{H}^{i} E_{H}^{i} \\
\frac{d I_{H}^{i}}{d t} & =\alpha_{H}^{i} E_{H}^{i}-\gamma_{H}^{i} I_{H}^{i}-\mu_{H}^{i} I_{H}^{i} \\
\frac{d R_{H}^{i}}{d t} & =\gamma_{H}^{i} I_{H}^{i}-\mu_{H}^{i} R_{H}^{i} \\
\frac{d S_{V}^{i}}{d t} & =\nu_{V}^{i} N_{V}^{i}-\lambda_{V}\left(N_{V}^{i} / N_{H}^{i}\right) S_{V}^{i}-\mu_{V}^{i} S_{V}^{i} \\
\frac{d E_{V}^{i}}{d t} & =\lambda_{V}^{i}\left(N_{V}^{i} / N_{H}^{i}\right) S_{V}^{i}-\alpha_{V}^{i} E_{V}^{i}-\mu_{V}^{i} E_{V}^{i} \\
\frac{d I_{V}^{i}}{d t} & =\alpha_{V}^{i} E_{V}^{i}-\mu_{V}^{i} I_{V}^{i}
\end{aligned}
$$

where:

- $i=1$ corresponds to the patch in Rio de Janeiro and $i=2$ the patch in Miami, Florida.

- The $\nu$ 's indexed by $V, H$ and the patch number are the birth rates. 
- The $\mu$ 's are the death rates.

- The $\alpha$ 's are the rates at which the exposed become infective.

- The $\gamma$ 's are the rates at which the infected recover.

- The density dependent $\lambda_{H}^{i}\left(N_{V}^{i} / N_{H}^{i}\right)$ and $\lambda_{V}^{i}\left(N_{V}^{i} / N_{H}^{i}\right)$ are the forces of infection from mosquitoes to humans and from humans to mosquitoes, respectively.

The force of infection from mosquitoes to humans is

$$
\lambda_{H}^{i}\left(N_{V}^{i} / N_{H}^{i}\right)=b_{H}^{i}\left(N_{V}^{i} / N_{H}^{i}\right) \frac{I_{V}^{i}}{N_{V}^{i}} \beta_{H}^{i}
$$

where $b_{H}^{i}\left(N_{V}^{i} / N_{H}^{i}\right)$ is the density-dependent average number of bites on a human by mosquitoes per unit time, explained in Section 2.1; $\frac{I_{V}^{i}}{N_{V}^{i}}$ is the probability that a mosquito is infectious ${ }^{1}$; and $\beta_{H}^{i}$ is the probability that a bite from an infected mosquito to a susceptible human will result in the transmission of the virus.

Similarly, the force of infection by humans to mosquitoes, $\lambda_{V}^{i}\left(N_{V}^{i} / N_{H}^{i}\right)$, is the product

$$
\lambda_{V}^{i}\left(N_{V}^{i} / N_{H}^{i}\right)=b_{V}^{i}\left(N_{V}^{i} / N_{H}^{i}\right) \frac{I_{H}^{i}}{N_{H}^{i}} \beta_{V}^{i}
$$

where $b_{V}^{i}\left(N_{V}^{i} / N_{H}^{i}\right)$ is the density-dependent average number of bites per mosquito on humans per unit time, explained in Section $2.1 ; \frac{I_{H}^{i}}{N_{H}^{i}}$ is the probability a human is infectious; and $\beta_{V}^{i}$ is the probability that a bite on an infected human by a susceptible mosquito will result in the transmission of the virus.

\footnotetext{
${ }^{1}$ We make the standard assumption that all biting mosquitoes have the same uniform probability $\frac{I_{V}^{i}}{N_{V}^{i}}$ of being infectious.
} 


\section{Humans}

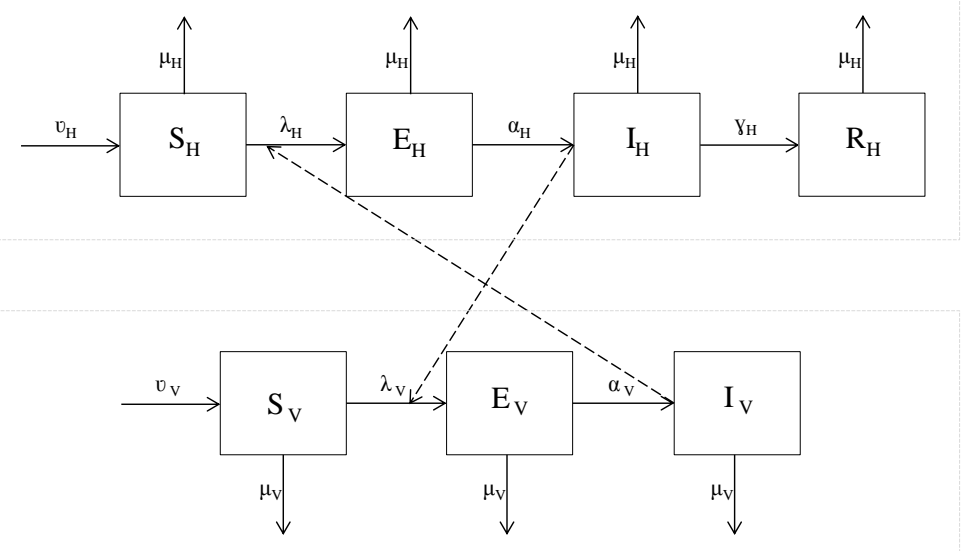

Vectors

Figure 1: Model used for transmission dynamics. Solid arrows indicate movement between compartments and dotted arrows represent bites by infected vectors (mosquitoes) on susceptible humans and bites on infected humans by susceptible vectors which can result in transmission of the virus. Figure modeled after those in $[14]$ and $[16]$.

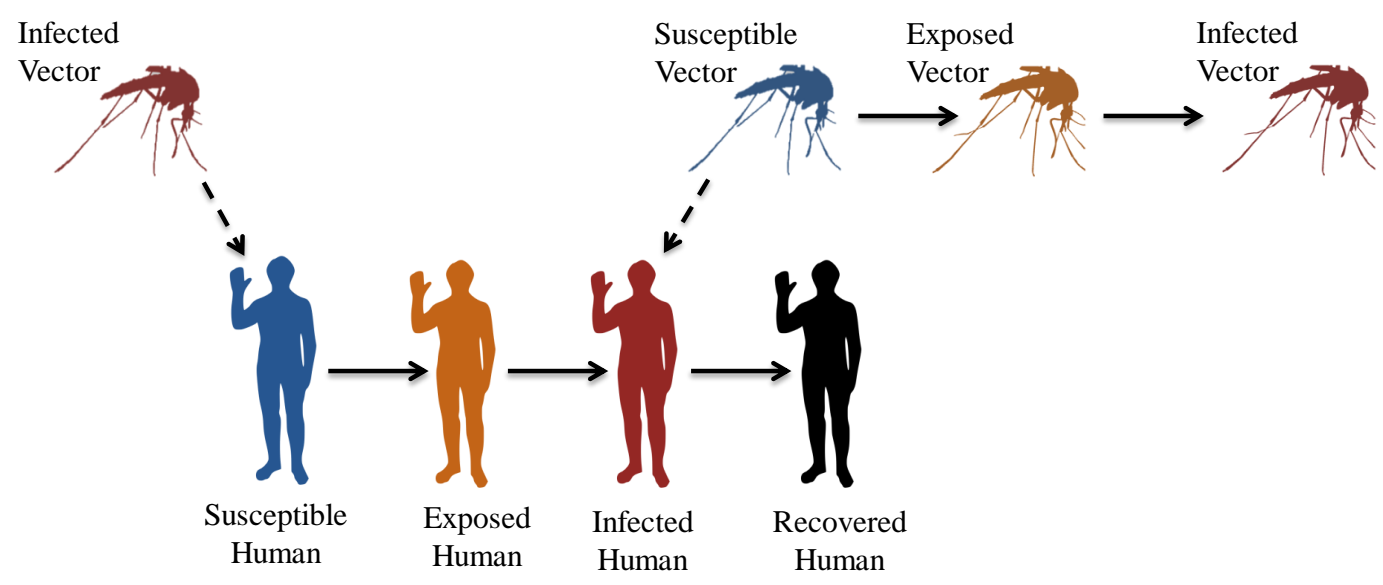

Figure 2: Transmission of Zika virus from an infected vector (mosquito) to a susceptible human and back to a susceptible vector. Dotted arrows indicate bites while solid arrows indicate a transfer between compartments

\subsection{Bite rates}

The bite rates $b_{H}^{i}\left(N_{V}^{i} / N_{H}^{i}\right)$ and $b_{V}^{i}\left(N_{V}^{i} / N_{H}^{i}\right)$ must satisfy the consistency constraint:

$$
b_{H}^{i} N_{H}^{i}=b_{V}^{i} N_{V}^{i}
$$

which reflects the equality between the total number of bites by mosquitoes on humans $\left(b_{H}^{i} N_{H}^{i}\right)$ and the total number of bites on humans by mosquitoes $\left(b_{V}^{i} N_{V}^{i}\right)$. We define $b_{H}^{\max , i}$ as the maximum number of times a 
human can be bitten per unit of time and $b_{V}^{\max , i}$ as the maximum number of times a mosquito can bite per unit of time $[7,16]$. Suboptimal biting rates are given in [7]. Here we propose optimal biting rates given by:

$$
\begin{gathered}
b_{H}^{i}\left(N_{V}^{i} / N_{H}^{i}\right)= \begin{cases}\frac{b_{V}^{\max , i} N_{V}^{i}}{N_{H}^{i}} & \text { if } \frac{N_{V}^{i}}{N_{H}^{i}} \leq \frac{b_{H}^{\max , i}}{b_{V}^{\text {max }, i}} \\
b_{H}^{\max , i} & \text { if } \frac{N_{V}^{i}}{N_{H}^{i}}>\frac{b_{H}^{\max , i}}{b_{V}^{\max , i}}\end{cases} \\
b_{V}^{i}\left(N_{V}^{i} / N_{H}^{i}\right)= \begin{cases}b_{V}^{\max , i} & \text { if } \frac{N_{V}^{i}}{N_{H}^{i}} \leq \frac{b_{H}^{\max , i}}{b_{V}^{\max , i}} \\
\frac{b_{H}^{\max , i} N_{H}^{i}}{N_{V}^{i}} & \text { if } \frac{N_{V}^{i}}{N_{H}^{i}}>\frac{b_{H}^{\max , i}}{b_{V}^{\max , i}} .\end{cases}
\end{gathered}
$$

These biting rates are optimal in the sense that when the relative number of vectors (mosquitoes) is small $\left(\frac{N_{V}^{i}}{N_{H}^{i}} \leq \frac{b_{H}^{\max , i}}{b_{V}^{\max , i}}\right)$ they can bite the maximum number of humans $\left(b_{V}^{\max , i},(12 \mathrm{a})\right)$ with a number of bites on each human (11a) smaller than the maximum $b_{H}^{\max , i}$ (the reasoning is flipped when the relative number of vectors is large). In all cases the biting rates are bounded by their maximum $b_{V}^{\max , i}$ and $b_{H}^{\max , i}$ (Figure 3 ).

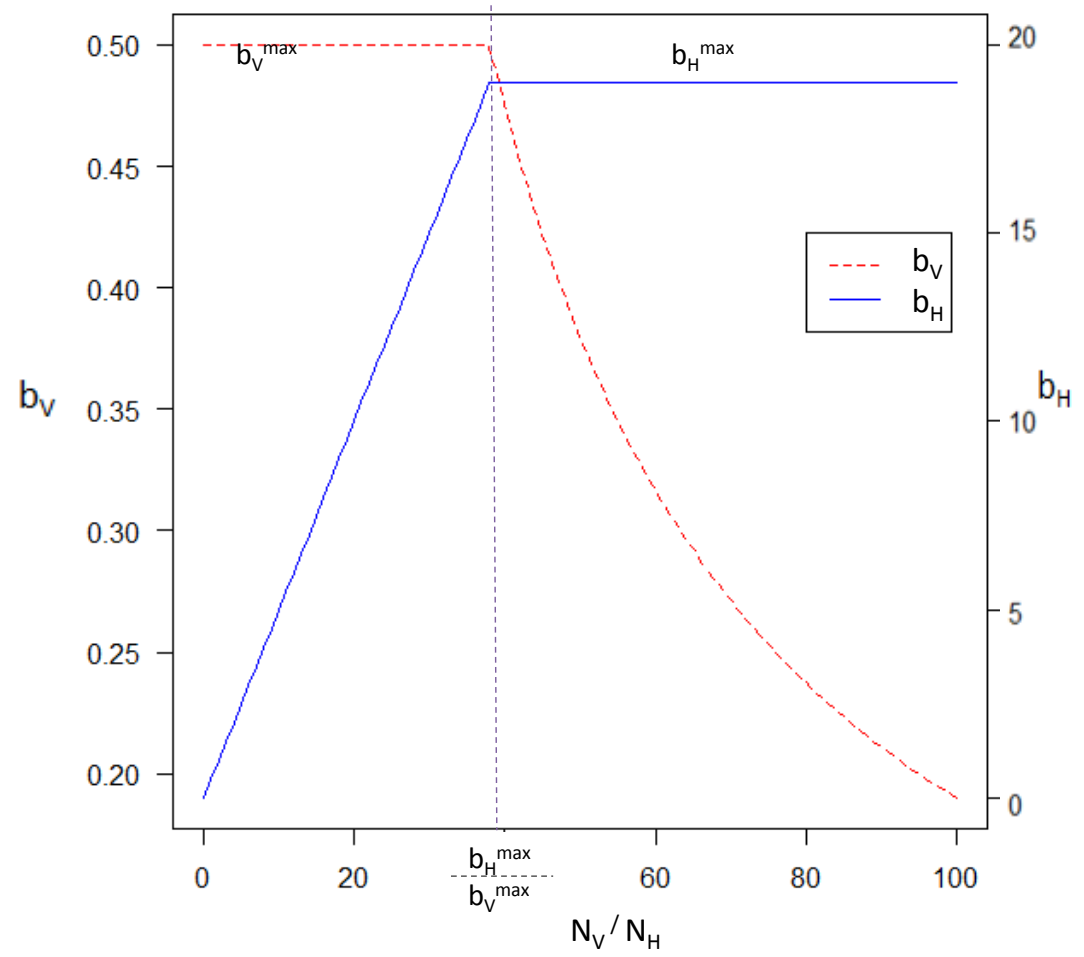

Figure 3: With patch indices omitted for clarity, optimal biting rates $b_{V}\left(N_{V} / N_{H}\right)$ and $b_{H}\left(N_{V} / N_{H}\right)$ as functions of the density $N_{V} / N_{H}$ (Eqs. (11)- (12)); $b_{V}\left(N_{V} / N_{H}\right)$ is at its maximum value for $N_{V} / N_{H}$ below $b_{H}^{\max } / b_{V}^{\max }$ and $b_{H}\left(N_{V} / N_{H}\right)$ is at its maximum value for $N_{V} / N_{H}$ above $b_{H}^{\max } / b_{V}^{\max }$. The numerical values are from Table 1. 


\section{$2.2 \quad$ Vector death rate}

Vector (mosquito) populations are lower during the winter. To capture the resulting counter-cyclical effect between the Northern and Southern hemispheres we created time-dependent cyclical death rates modeled after [3]. These death rates, $\mu_{V}^{i}(t)$ for patches $i=1,2$, yield maximum death rates during each hemisphere's winter:

$$
\begin{aligned}
& \mu_{V}^{1}(t)=\frac{1}{2}\left[\left(\mu_{V, \text { min }}^{1}-\mu_{V, \text { max }}^{1}\right) \sin \left(\frac{2 \pi t}{365}\right)+\mu_{V, \text { min }}^{1}+\mu_{V, \text { max }}^{1}\right] \\
& \mu_{V}^{2}(t)=\frac{1}{2}\left[\left(\mu_{V, \text { min }}^{2}-\mu_{V, \text { max }}^{2}\right) \sin \left(\frac{2 \pi(t-183)}{365}\right)+\mu_{V, \text { min }}^{2}+\mu_{V, \text { max }}^{2}\right]
\end{aligned}
$$

The average birth rate $\left(\nu_{V}^{i}\right)$ is the midpoint $\left(\mu_{V, \min }^{i}+\mu_{V, \max }^{i}\right) / 2$ between the maximum and minimum death rates to ensure a stable mosquito population in the long run. The variable $t$ symbolizes the number of days which have passed since the beginning of the cycle, set to September 1, 2015. The resulting mosquito populations for the two patches are plotted in Figure 4.

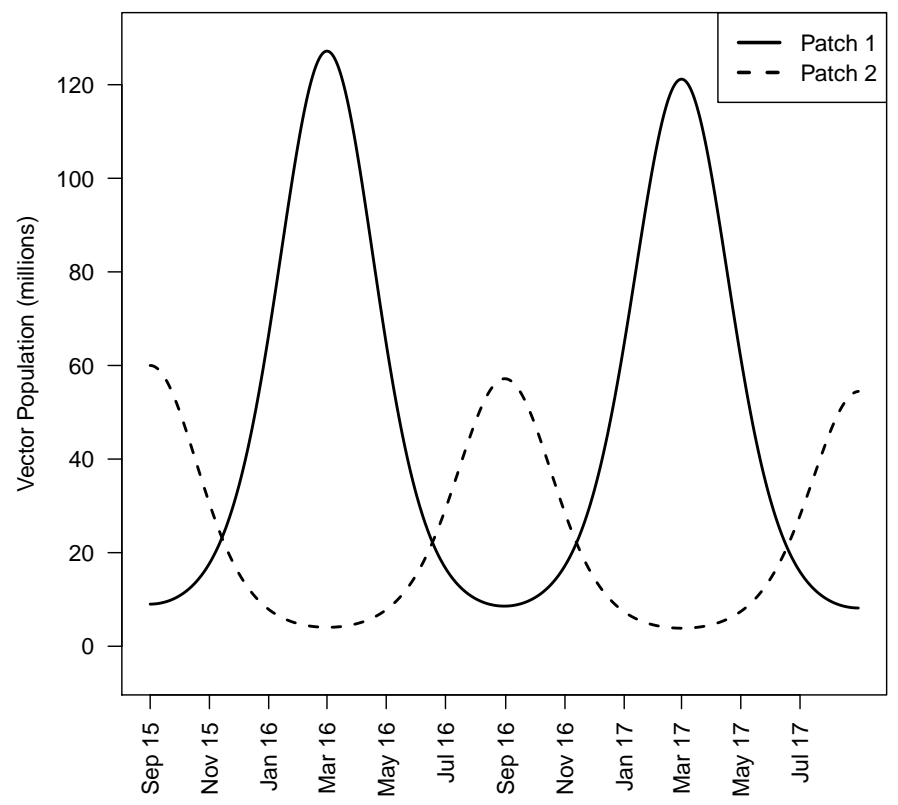

Figure 4: Cyclical vector (mosquito) population in Patches 1 (Rio de Janeiro) and 2 (Miami) resulting from death rates in Eqs. (13) and (14). The populations reach their maximum roughly during each hemisphere's summer. The numerical values are from Table 1.

\subsection{Basic Reproduction Number}

The basic reproduction number, $R_{0}^{i}(t)$, is the time-dependent number of new human infections caused by one exposed human via the mosquitoes in a disease-free population (Figure 5). This number varies because the 
mosquito population, $N_{V}^{i}(t)$, is seasonal. $R_{0}^{i}(t)$ is the product:

$$
R_{0}^{i}(t)=R_{H V}^{i}(t) R_{V H}^{i}(t)
$$

where $R_{H V}^{i}(t)$ is the number of new mosquito infections caused by the one exposed human and $R_{V H}^{i}(t)$ is the number of secondary infections in humans caused by one of the infected mosquitoes. In our model

$$
R_{H V}^{i}(t)=\frac{\alpha_{H}^{i}}{\alpha_{H}^{i}+\mu_{H}^{i}} b_{H}^{i}\left(N_{V}^{i}(t) / N_{H}^{i}(t)\right) \beta_{V}^{i} \frac{1}{\gamma_{H}^{i}+\mu_{H}^{i}}
$$

where the first term in the product is the probability of an exposed human reaching the infected state; the second term is the number of vectors (mosquitoes) that bite the infected human; the third term is the probability that the bite will transmit the pathogen; the fourth term is the average time spent in the infected stage.

Similarly for vectors (mosquitoes) we have

$$
R_{V H}^{i}(t)=\frac{\alpha_{V}^{i}}{\alpha_{V}^{i}+\mu_{V}^{i}} b_{V}^{i}\left(N_{V}^{i}(t) / N_{H}^{i}(t)\right) \beta_{H}^{i} \frac{1}{\mu_{V}^{i}} .
$$

In the standard case of constant mosquito and human populations the basic reproduction numbers would be constants $R_{0}^{i}, i=1,2$. For $R_{0}^{i}>1$ an outbreak occurs, while for $R_{0}^{i}<1$ it does not. Here a timedependent $R_{0}^{i}(t)$ larger than one is merely an indication of a potential outbreak, were the human and mosquito populations to remain constant after time $t$. Similarly, $R_{0}^{i}(t)<1$ indicates there would be no outbreak with unchanging populations. 

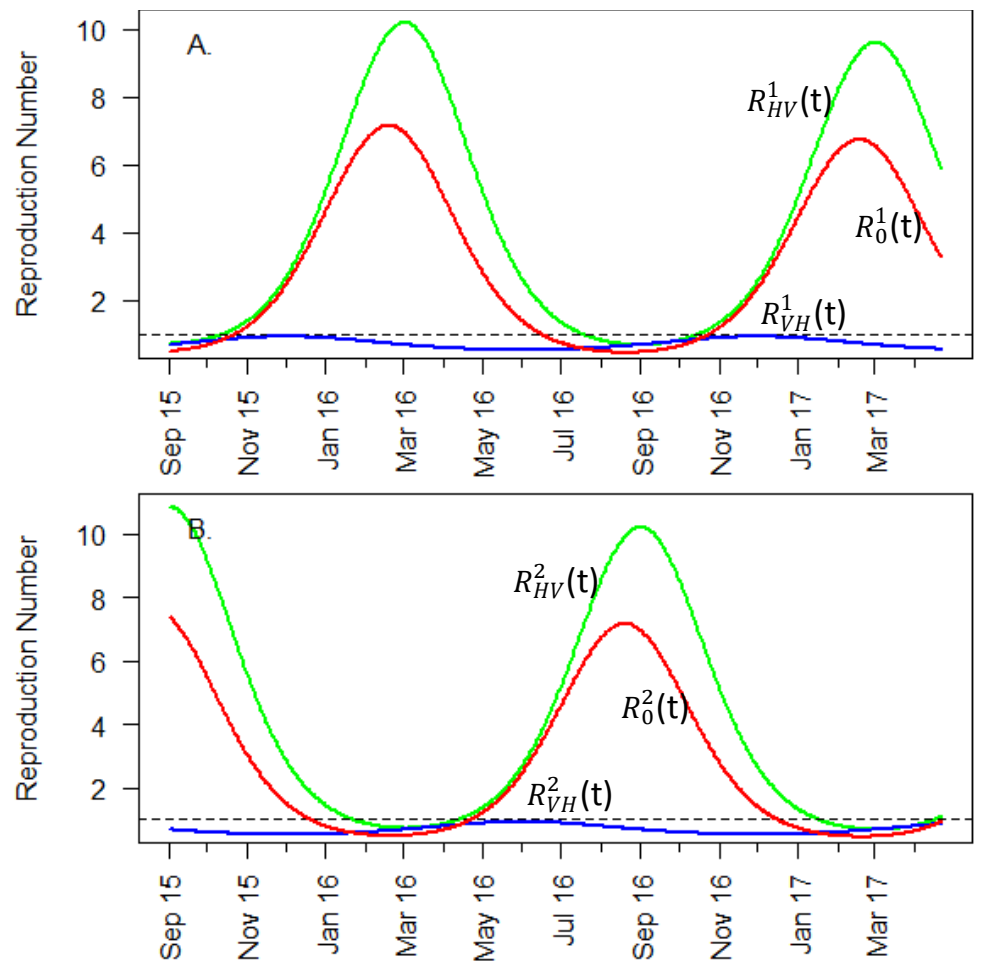

Figure 5: Plot for both patches of $R_{H V}^{i}(t), R_{V H}^{i}(t)$ and resulting basic reproduction numbers $R_{0}^{i}(t)$ from Eq. (15). These plots show the counter-cyclical conditions in both hemispheres with the $R_{0}^{i}(t)$ peaking in February for Patch 1 and in August for Patch 2. Figure 5.A applies to Patch 1 and Figure 5.B is for Patch 2 . The black dotted line at 1 to show when the basic reproduction number $R_{0}^{i}(t)$ is below or above 1 . The numerical values are from Table 1.

\section{Application of the Model}

The goal of this paper is to investigate whether visitors from Miami (Patch 2) to either Carnival in February 2016 or the Olympics in August 2016 can be infected in Rio de Janeiro (Patch 1) and bring the Zika virus back. We will explore these possibilities by having a group of visitors moving from Miami to Rio de Janeiro for these events and back at their conclusion. Parameter values, taken from the literature, are given in Table 1. We make the assumption that the parameters applied to the general population also apply to the visitors. 
Table 1: Parameters for the model. Rates are given in units of days ${ }^{-1}$ and durations are in days.

\begin{tabular}{|c|c|c|c|}
\hline Parameter & Definition & Baseline & Source \\
\hline \multicolumn{4}{|c|}{ Human Population } \\
\hline$\nu_{H}$ & Human birth rate & $1 / 19,210$ & {$[6]$} \\
\hline$\mu_{H}$ & Human death rate & $1 / 45,625$ & [6] \\
\hline $1 / \alpha_{H}$ & Human incubation period & 5.9 & [14] \\
\hline $1 / \gamma_{H}$ & Human infectious period & 5.9 & {$[15]$} \\
\hline$\beta_{H}$ & Vector (Mosquito) to Human transmission probability & 0.42 & [14] \\
\hline$b_{H}^{\max }$ & Max bites per human & 19 & {$[8]$} \\
\hline \multicolumn{4}{|c|}{ Vector (Mosquito) Population } \\
\hline$\nu_{V}$ & Vector (Mosquito) birth rate & $1 / 7.8$ & [14] \\
\hline$\mu_{V, \min }$ & Vector (Mosquito) minimum death rate & $1 / 9.3$ & \\
\hline$\mu_{V, \max }$ & Vector (Mosquito) maximum death rate & $1 / 6.5$ & \\
\hline $1 / \alpha_{V}$ & Vector (Mosquito) incubation period & 10.5 & [2] \\
\hline$\beta_{V}$ & Human to Vector (Mosquito) transmission probability & 0.42 & {$[14]$} \\
\hline$b_{V}^{\max }$ & Max bites by Vector (Mosquito) & 0.5 & {$[18,22]$} \\
\hline \multicolumn{4}{|c|}{ Initial Values } \\
\hline \multicolumn{4}{|c|}{ i. Patch 1: Rio de Janeiro } \\
\hline$S_{H}^{1}$ & Susceptible Humans & $12,902,000$ & {$[6]$} \\
\hline$E_{H}^{1}$ & Exposed Humans & $129,020^{2}$ & \\
\hline$I_{H}^{1}$ & Infected Humans & 0 & \\
\hline$R_{H}^{1}$ & Recovered Humans & 0 & \\
\hline$S_{V}^{1}$ & Susceptible Vectors (Mosquitoes) & $9,000,000^{3}$ & \\
\hline$E_{V}^{1}$ & Exposed Vectors (Mosquitoes) & 0 & \\
\hline$I_{V}^{1}$ & Infected Vectors (Mosquitoes) & 0 & \\
\hline \multicolumn{4}{|c|}{ ii. Patch 2: Miami } \\
\hline$S_{H}^{2}$ & Susceptible Humans & $5,817,000$ & {$[6]$} \\
\hline$E_{H}^{2}$ & Exposed Humans & 0 & \\
\hline$I_{H}^{2}$ & Infected Humans & 0 & \\
\hline$R_{H}^{2}$ & Recovered Humans & 0 & \\
\hline$S_{V}^{2}$ & Susceptible Vectors (Mosquitoes) & $60,000,000^{4}$ & \\
\hline$E_{V}^{2}$ & Exposed Vectors (Mosquitoes) & 0 & \\
\hline$I_{V}^{2}$ & Infected Vectors (Mosquitoes) & 0 & \\
\hline
\end{tabular}

\subsection{Data fitting for Patch 1}

We fit the model for Patch 1 to the bell-shaped pattern of cases for Brazil collected by the Pan American Health Organization [17], assuming that the pattern applied for Rio de Janeiro as well. For the model in Patch 1 we take into account the visitors to Patch 1 for either Carnival or the Olympics (described in Section

\footnotetext{
${ }^{2}$ We made a somewhat arbitrary assumption that at the start of our model (September 1, 2015) there would only be exposed humans and no infected humans or exposed/infected vectors. The number of exposed humans $\left(E_{H}^{1}\right)$ was chosen through trial and error as a percentage of the susceptible human population in Patch 1 . We found that with a 1\% exposed population (129,020) the model for the patch roughly resembles the observed pattern for the number of new infections during the first half of 2016 in Brazil [17].

${ }^{3}$ Our model starts on September 1, 2015 when the number of vectors (mosquitoes) in Rio de Janeiro is at a minimum. The estimated 9,000,000 was chosen so that the maximum susceptible vector population (130,000,000, Figure 4) reached around March would be roughly ten times higher than the population of Rio de Janeiro based on the data in [16]. By doing this the model values for Patch 1 resemble the pattern in [17].

${ }^{4}$ We made an assumption that the same ratio of roughly ten vectors (mosquitoes) to one human during peak vector season would apply for Miami as Rio de Janeiro. The September 1st start date of the model is during peak mosquito season in Miami and so $60,000,000$ is the maximum number of mosquitoes there.
} 
3.2). Our model shows an outbreak between January and April 2016 and therefore has a shorter duration than the pattern presented in [17] with the primary outbreak between January and April but a continuation of a small number of cases into June 2016. While the model does not capture the longer outbreak of the disease it accurately represents the peak in February 2016 (Figure 6.A). It also predicts that 100\% of the population will be infected which is on the high side but in general agreement with the model in [14] for the Zika outbreak in French Polynesia in 2013-2014 where it was estimated that 94\% of the population there had become infected.

We did not compare the numerical results of our model with actual data because reporting on Zika in Brazil did not start until January 2016 and there was not specific data on the number of cases in Rio de Janeiro. There could be a great deal of under-reporting of cases since up to $80 \%$ of cases are asymptomatic [5]. Also, during the French Polynesia outbreak it was predicted only $11.5 \%$ of infections were reported [14] and while there is perhaps better surveillance in Brazil it is probably not complete.

With the assumption that once a person recovers from the disease they are immune, an outbreak in Rio de Janeiro is unlikely to occur again until there is a significant susceptible population or a different strain of Zika virus emerges for which the population is not immune.

\subsection{Modeling the Visitors to Patch 1}

To model the visitors to either Carnival or the Olympics a group of 100 people was subtracted on either February 3rd or August 3rd from Patch 2 in Miami and added to Patch 1 in Rio de Janeiro. To keep track of the visitors, a sub-patch in Rio de Janeiro was used that only included the visitors from Miami but allowed interactions with humans and mosquitoes in Rio de Janeiro. On February 12th or August 21st the visitors (now susceptible, exposed, infected, or recovered) in the sub-patch were moved back to the general population of Patch 2. The dates for the events were chosen with two extra days before the event occurs and one extra day after to include the added time that people are likely to spend in Rio de Janeiro. To see the effects individually we modeled visitors to either Carnival or the Olympics and did not look at the combined effect.

\subsubsection{Visitors to Carnival}

Visitors to Carnival arrived during the month of February where there was a peak number of Zika cases recorded in Brazil [17]. This is shown in Figure 6.A. Visitors during Carnival between February 3rd and 12th 2016 are therefore at the highest risk of contracting the disease compared to visitors during other times. If the same parameters (Table 1) are used for both patches (Rio de Janeiro and Miami) the model shows that the virus can be brought to the United States where it will remain at a low level until the peak of the mosquito population in Miami in September (Figure 4). This will then cause an outbreak in Miami between October and December 2016 resulting in roughly $75 \%$ of the population becoming infected (Figure 6.B). 
These results are consistent with the values of the time-dependent basic reproduction numbers $R_{0}^{i}(t)$ over the year (Figure 5). Indeed, Figure 5.A shows that $R_{0}^{1}(t)$ stays above 7 during Carnival in Rio de Janeiro. This high number indicates visitors are likely to be infected and bring the disease back to Miami. Once back in Miami, the virus is maintained at a low level until the mosquito population (Figure 4) picks up and the basic reproduction number $\left(R_{0}^{2}(t)\right)$ in Miami reaches 6 in October (Figure 5.B). Following this, an outbreak occurs in Miami during the fall of 2016 (Figure 6.B).
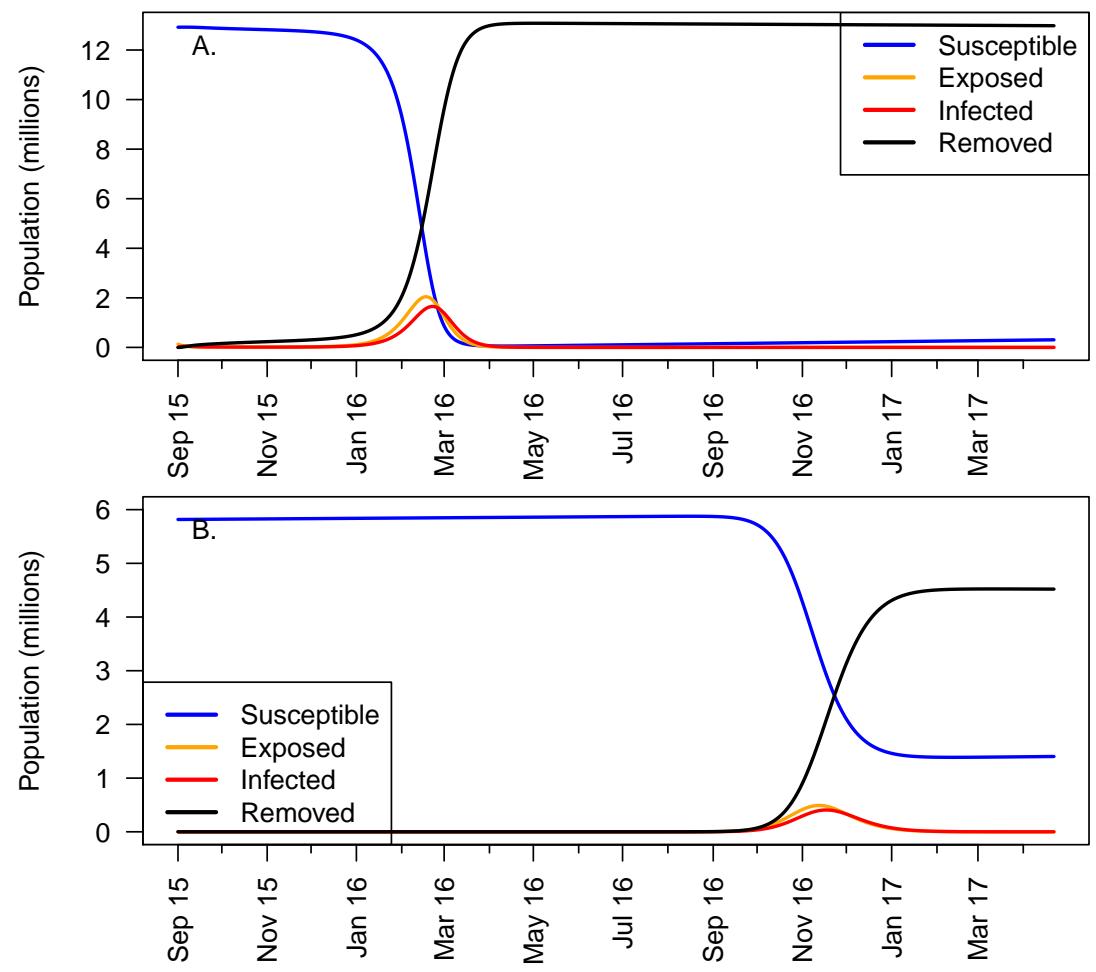

Figure 6: Modeled population in each compartment in Rio de Janeiro (Figure 6.A) and Miami with visitors who were in Rio de Janeiro for Carnival (Figure 6.B). There is no visible effect on the plots when the visitors leave or return from Carnival because of the small numbers.

\subsubsection{Visitors to the Olympics}

By August 2016 the model predicts that the virus has already infected the majority of the human population in Patch 1 and that there will be a low mosquito population (Figure 4). Due to both of these factors Zika virus is no longer being transmitted in Rio de Janeiro during the Olympics (Figure 7.A). The model shows that visitors present August 3-21, 2016 in Rio de Janeiro do not become infected and will not cause an outbreak in Miami (Figure 7.B) even though conditions are perfect there for an outbreak in August $\left(R_{0}^{2}(t)>7\right.$, Figure 5.B). 

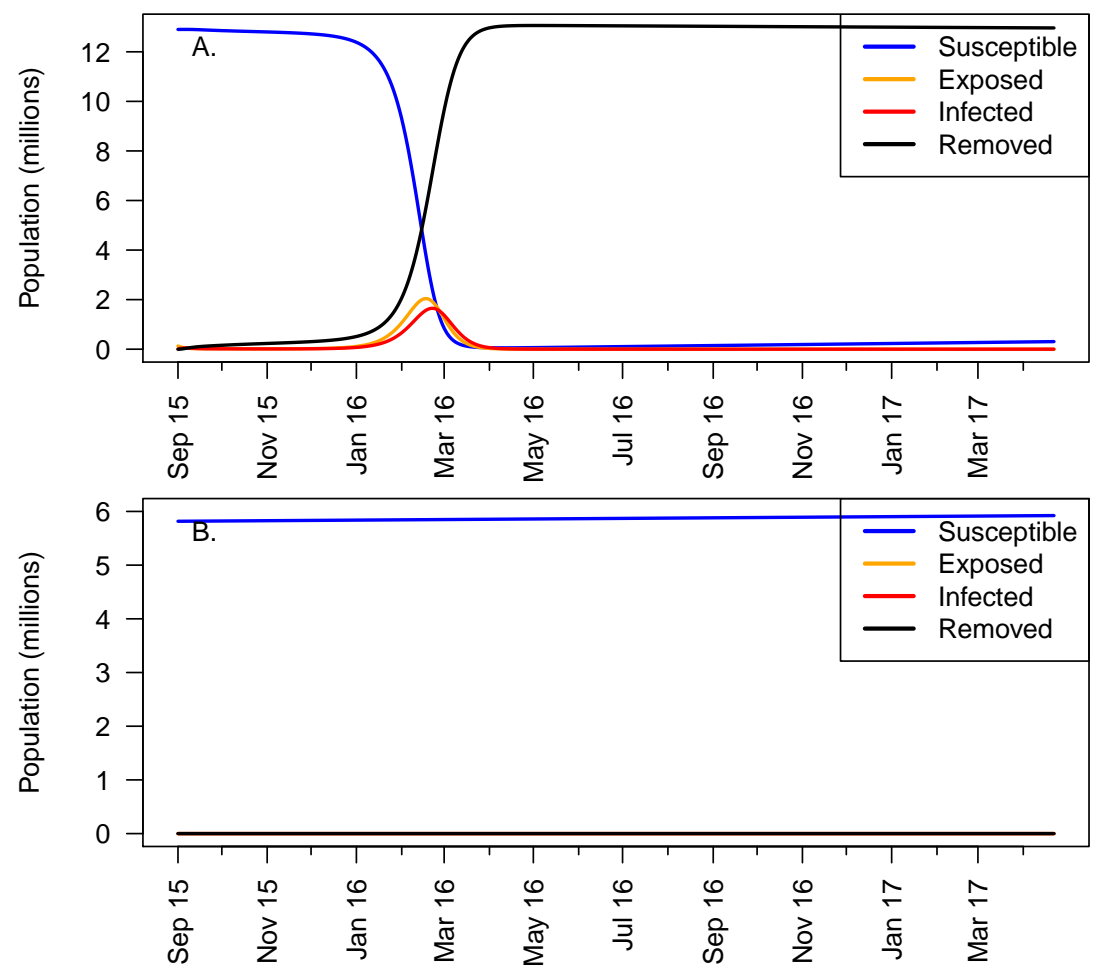

Figure 7: Modeled population in each compartment for Rio de Janeiro (Figure 7.A) and Miami with visitors who were in Rio de Janeiro for the Olympics (Figure 7.B). There is no visible effect on the plots when the visitors leave or return from the Olympics because of the small numbers.

\subsection{Sensitivity Analysis}

\subsubsection{Change in bite rates}

The results of Sections 3.2.1 and 3.2.2 assume that the parameters (Table 1) are the same for both patches. However, this may not be the case because mosquito populations [13] and lifestyles are different in Miami and in Rio de Janeiro. We will assess the sensitivity of the model to variations in two important parameters which reflect these differences: i) the maximum number of bites per mosquito on humans per day $\left(b_{V}^{\max , i}\right)$ and ii) the maximum number of bites on a human by mosquitoes per day $\left(b_{H}^{\max , i}\right)$. We do this only for Patch 2 because if these parameters are modified in Patch 1 the model no longer follows the disease outbreak pattern presented in [17].

If the baseline value $b_{V}^{\max , 2}$ is reduced by $10 \%$ from 0.5 [16] to 0.45 the percentage infected in Miami by visitors to Carnival drops from $75 \%$ (Figure 6.B) to $15 \%$ (Figure 8.A). However $b_{V}^{\max , 2}$ may be even smaller than 0.45. Indeed, if we take an average value of $b_{V}^{\max , 2}$ for Aedes aegypti $(0.5,[16])$ and Aedes albopictus $(0.26,[16])$ to capture the presence of both species of mosquitoes in Miami we obtain $0.38=(0.5+0.26) / 2$. This value is within the accepted broad range of 0.33 to 1 for Aedes aegypti $[22,18]$ and 0.19 to 0.39 for 
Aedes albopictus $[21,9]$. With this value, there is no predicted outbreak in Miami due to visitors to Carnival (Figure 8.B). Regardless of the value for $b_{V}^{\max , 2}$, the visitors to the Olympics do not start an outbreak in Miami because the model predicts the virus is not present in Rio de Janeiro in August 2016.
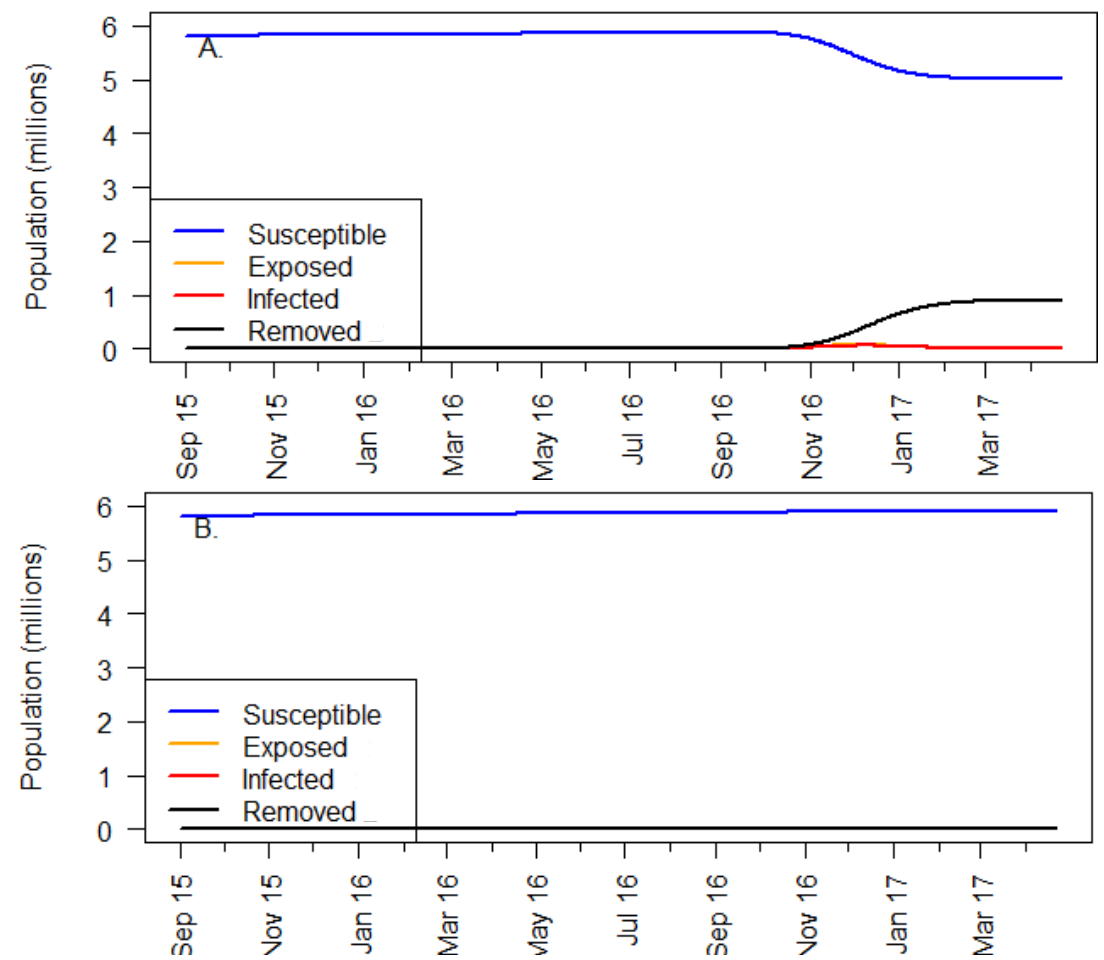

Figure 8: Modeled populations in Patch 2 due to visitors for Carnival for $b_{V}^{\max , 2}=0.45$ (Figure 8.A) and $b_{V}^{\max , 2}=0.38$ (Figure 8.B). See text for discussion.

The model is not as sensitive to changes in the maximum number of bites on a human $\left(b_{H}^{\max , 2}\right)$. Indeed to reduce the percentage infected in Miami from $75 \%$ to $15 \%$ (as was seen when $b_{V}^{\max , 2}$ was reduced by $10 \%$ (Figure 8.A)) we need to reduce $b_{H}^{\max , 2}$ by over $80 \%$ from 19 to 3.1. For there to be no outbreak due to a change in $b_{H}^{\max , 2}$ from the visitors to Carnival the $b_{H}^{\max , 2}$ must be less than 2.1. This falls in the range of 0.1 to 50 presented by $[8]$ and is therefore not unreasonable.

\subsubsection{Change in number of visitors}

In the results described in Sections 3.2.1 and 3.2.2 100 visitors were presumed to go to Rio de Janeiro from Miami during either Carnival or the Olympics. However, the actual number of visitors to this event is difficult to estimate because only the total number of foreign visitors to Carnival is recorded [20]. To reflect this, we looked at how the number of visitors affects the outbreak size in Miami (with the same parameters in each patch). Regardless of the number of visitors from Miami to the Olympics they will not cause an outbreak upon their return because there is no outbreak in Rio de Janeiro in August. 
However, the size of the outbreak that occurs in Miami due to visitors to Carnival will depend on the number of visitors. For example, if the number of visitors is reduced ten times (from 100 to 10) the model shows a smaller outbreak in Miami with less than 35\% infected (Figure 9.A), instead of $75 \%$ (Figure 6.B). However, if there are ten times more visitors (1000) the model suggests a much larger outbreak with over $90 \%$ of the population becoming infected in Miami (Figure 9.B).
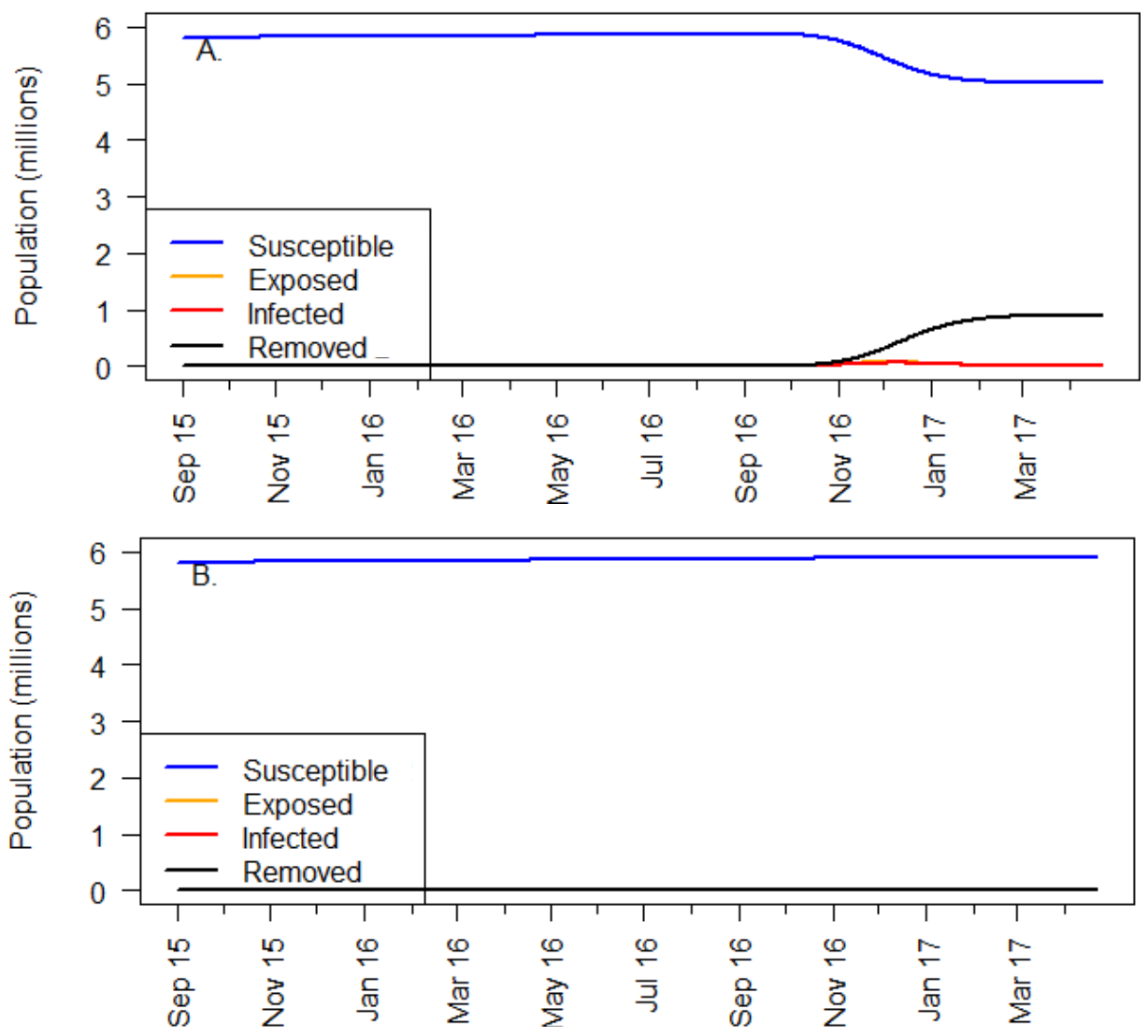

Figure 9: Modeled populations in Patch 2 due to visitors for Carnival for 10 (Figure 9.A) and 1000 visitors (Figure 9.B). See text for discussion.

\section{Discussion and Conclusion}

We created a model for the spread of Zika virus in Rio de Janeiro which reproduced the pattern of Zika virus disease spread in Brazil [17]. With this model we investigated if visitors to Rio de Janeiro for either Carnival in February 2016 or the Olympics in August 2016 would bring the virus back to Miami and spread it throughout the population there. The model shows that if the parameters are the same in Miami and Rio de Janeiro, a small population of visitors to Carnival can bring back the Zika virus and cause an outbreak that infects $75 \%$ of the population in Miami during the fall of 2016 . However, the model suggests that by August 2016 the virus spread has already peaked [17] and visitors to the Olympics will not be exposed and 
cause an outbreak in Miami.

To analyze the sensitivity of our model we looked at how the sized of the outbreak in Miami can be affected by different mosquito populations, an indoor lifestyle of humans in Miami, and the number of visitors to Rio de Janeiro. To account for a different mosquito population, one dominated by Aedes albopictus instead of Aedes aegypti we reduced the maximum bites per mosquito on humans $\left(b_{V}^{\max , 2}\right)$ by $10 \%$. This causes the percentage infected in Miami to drop from $75 \%$ to $15 \%$. However, to achieve the same reduction to $15 \%$ the maximum bites per human by mosquitoes $\left(b_{H}^{\max , 2}\right)$ needs to be decreased by over $80 \%$ by using, for example, air conditioning or window screens. A small outbreak in Miami is consistent with the history of Miami where there have been no outbreaks of dengue or chikungunya (spread by the same vectors) on the scale seen in Rio de Janeiro [3].

We also analyzed how the number of visitors to Rio de Janeiro for Carnival affects the size of the outbreak in Miami. If the number of visitors is reduced to 10, the outbreak in Miami infects $35 \%$ of the population compared to $75 \%$ (with 100 visitors). However, if the number of visitors is increased to 1000 there is an outbreak that infects $90 \%$ of the population of Miami. The model's predictions of infected percentages may be on the high side. This is perhaps due to the homogeneous mixing assumptions or because of imprecision in the parameter values. Also, no assumptions were made regarding quarantine, education, or other public or individual intervention measures. This sensitivity analysis shows that limiting exposure to the mosquitoes and reducing the number of visitors to Rio de Janeiro decreases the size of a secondary outbreak in Miami.

In all cases, regardless of the number of visitors or the parameters, the model predicts that visitors for the Olympics are not exposed and will not cause an outbreak in Miami. This is in agreement with WHO's dismissal of claims that the Olympics should be moved or postponed [23].

We did not conduct an equilibrium analysis of our model for two reasons. First, the assumption that people are immune once they recover means this is a one-cycle disease which obviates the need for an equilibrium analysis. Second, the exogenously changing mosquito death rate would make such an analysis considerably more complicated.

This model only considered visitors from Miami and not the total expected number of around 500,000 coming from many different countries for either Carnival or the Olympics. This would require as many patches as places of origin and would make the model intractable and beyond the scope of this paper. This paper should be taken as a simplified study of the effect of a mass gatherings on the spread of Zika virus between South and North America and not as a firm predictor of Zika's presence or absence in Miami. 


\section{References}

[1] A. ATtARAn, A. CAPLAN, C. GAFFnEy, and L. IGEL, Rio Olympics Later, retrieved from https://rioolympicslater.org (2016).

[2] J. BOORMAN and J. PORTERFIELD, A simple technique for infection of mosquitoes with viruses transmission of Zika virus, Trans. Roy. Soc. Trop. Med. Hyg., 50(1956), pp. 238-242.

[3] M. N. BURATtini, F. A. B. COUTINHO, L. F. LOPEZ, and A. WILDER-SMITH, Potential exposure to Zika virus for foreign tourists during the 2016 Carnival and Olympic Games in Rio de Janeiro, Brazil, Epid. Infec., 9(2016), pp. 1-3.

[4] M. CHAN, WHO Director-General summarizes the outcome of the Emergency Committee regarding clusters of microcephaly and Guillain-Barre syndrome, 2016.

[5] CENTERS FOR DISEASE CONTROL AND PREVENTION, Zika Virus, retreived from http://www.cdc.gov/zika/index.html, 2016.

[6] Central IntelligenCE AGENCY, The World Factbook, retrieved from https://www.cia.gov/library/publications/the-world-factbook/rankorder/2054rank.html , 2016.

[7] N. CHITNIS, J. CUSHING, and J. HYMAN, Bifurcation analysis of a mathematical model for maleria transmission, SIAM J. Appl. Math., 67(2006), pp. 24-45.

[8] N. CHITNIS, J. HYMAN, and J. CUSHING, Determining important parameters in the spread of malaria through the sensitivity analysis of a mathematical model, Bull. Math. Biol., 70(2008), pp. 1272-1296.

[9] H. DelatTe, G. GIMONnEAU, A. TRIBOIRE, and D. FONTEnILLE, Influence of temperature on immature development, survival, longevity, fucundity, and gonotrophic cycles of Aedes albopictus, vector of chikungunya and dengue in the Indian Ocean, J. Med. Entomol., 46(2009), pp. 33-41.

[10] GREATER MIAMI CONVENTION AND VISITORS BUREAU, Greater Miami and the Beaches 2010 Visitor Industry Overview, Miami, FL, 2011.

[11] W. O. KERMACK and A. G. McKENDRICK, A Contribution to the Mathematical Theory of Epidemics, Proc. Roy. Soc. Lond. A, 115(1927), pp. 700-721.

[12] M. K. KINDHAUSER, T. ALLEN, V. FRANK, R. S. SANTHANA, and C. DYE, Zika: the origin the spread of a mosquito-borne virus, Bull. World Health Organ., 2016.

[13] M. U. G. KRAEMER, M. E. SINKA, K. A. DUDA, A. Q. N MYLNE, F. M. SHEARER, C. M. BARKER, C. G. MOORE, R. G. CARVALHO, G. E. COELHO, W. V. BORTEL, G. HENDRICKX, F. SCHAFFNER, 
I. R. F. ELYAZAR, H. TENG, O. J. BRADY, J. P. MESSinA, D. M. PIGOTT, T. W. SCOTT, D. L. SMITH, G. R. W. WINT, N. GOLDING, and S. I. HAY, The global distribution of the arbovirus vectors Aedes aegypti and Ae. albopictus, eLife, 4(2015), retrieved from https://elifesciences.org/content/4/e08347.

[14] A. J. KUCHARSKI, S. FUNK, R. M. EGGO, H. MALLET, W. J. EDMUNDS, and E. J. NILLES, Transmission Dynamics of Zika Virus in Island Populations: A Modelling Analysis of the 2013-14 French Polynesia Outbreak, PLoS Negl. Trop. Dis., 10(2016).

[15] H. P. MALLET, A. L. VIAL, and D. MUSSO, Bilan de l'épidémie a virus ZIKA en Polynésie Francaise 2013-2014, Bull. Inform. Sanitaires, Epidemiologiques, et Statistiques, (2015).

[16] C. A. MANORE, K. S. HICKMANN, S. XU, H. J. WEARING, and J. M. HYMAN, Comparing dengue and chikungunya emergence and endemic transmission in A. aegypti and A. albopictus, J. Theoret. Biol., 356(2014), pp. 174-191.

[17] PAN AMERICAN HEALTH ORGANIZATION/WORLD HEALTH ORGANIZATION, Zika Epidemiological Update-30 June 2016, PAHO/WHO, Washington, D.C., 2016.

[18] J. L. PUTNAM and T. W. SCOTT, Blood feeding behavior of dengue-2 virus-infected Aedes aegypti, Am. J. Trop. Med. Hyg., 55(1995), pp. 225-227.

[19] S. ROMERO and R. R. RUIZ, Researchers Weigh Risks of Zika Spreading at Rio Olympics, New York Times, 2016.

[20] K. SILVER, Traveling to Rio for Carnival or the Olympics? Here's what you should know about the virus, Washington Post, 2016.

[21] M. SIVANATHAN, The Ecology and Biology of Aedes aegypti (1.) and Aedes albopictus (skuse)(diptera: Culicidae) and the Resistance Status of Aedes albopictus (field strain) against Organophosphates in Penang, Malaysia, Universiti Sains Malaysia, 2006.

[22] M. TRPIS and W. HAUSSERMANN, Dispersal and other population parameters of the Aedes aegypti in an African village and their possible significance in epidemiology of vector-borne diseases, Am. J. Trop. Med. Hyg., 35(1986), pp. 1263-1279.

[23] W.H.O. Dismisses Call to Move Olympics Over Zika Virus in Brazil, The New York Times, retrieved from http://www.nytimes.com/2016/05/29/world/americas/zika-olympics-rio-who.html (2016).

[24] W.H.O, Communicable disease alert and response for mass gatherings:key considerations, WHO, Geneva, Switzerland, 2008. 